\title{
Low Complexity Discrete Bit-Loading for OFDM Systems with Application in Power Line Communications
}

\author{
Khalifa S. Al-Mawali, Amin Z. Sadik, Zahir M. Hussain \\ School of Electrical and Computer Engineering, RMIT University, Melbourne, Australia \\ E-mail:k.almawali@student.rmit.edu.au,zmhussain@ieee.org \\ Received January 27, 2011; revised February 19, 2011; accepted March 14, 2011
}

\begin{abstract}
Adaptive bit-loading algorithms can improve the performance of OFDM systems significantly. The tradeoff between the performance of the algorithm and its computational complexity is essential for the implementation of loading algorithms. In this paper, we present a low complexity non-iterative discrete bit-loading algorithm to maximize the data rate subject to specified target BER and uniform power allocation. Simulation results show that the proposed algorithm outperforms the equal-BER loading and achieves similar rates to incremental allocation, yet with much lower complexity.
\end{abstract}

Keywords: Bit-Loading, OFDM, Power Line Communications

\section{Introduction}

Power Line Communication (PLC) technology has received a great amount of research interest in the last decade. This technology exploits the already existing and ubiquitous power line distribution infrastructure to provide a broadband multimedia connectivity solution to and within the home or office. Orthogonal Frequency Division Multiplexing (OFDM) has been a major candidate for PLC systems as well as many other broadband communication systems. This is mainly due to its robustness to multipath, selective fading and different kinds of interference. Examples of its applications include Digital Audio Broadcasting (DAB), Terrestrial digital TV (DVB-T), wireless LANs and Wi-Max.

In a conventional OFDM system, all subcarriers use a fixed constellation size. Therefore, their overall error probability is dominated by the subcarriers that have the worst signal-to-noise ratios (SNR). When channel state information is available, the performance of OFDM can be significantly improved by using adaptive modulation [1]. In adaptive modulation, different parameters including data rate, transmit power, instantaneous bit-error-rate (BER), constellation size and channel code or scheme can be adjusted according to the subchannel fading conditions.

Several bit/power loading algorithm can be found in the literature [2-15]. Most of these algorithms can be classified, based on their objective function, into two categories: margin-adaptive (MA) algorithms (e.g. [7-9]) that strive to minimize the transmitted power subject to data rate and BER constraints, and rate-adaptive (RA) algorithms (e.g. [10-14] that strive to maximize the data rate subject to power and BER constraints. In addition, some algorithms that have different objectives can also be found in the literature. For example, the algorithm proposed by Goldfeld et al. [12] is aimed at minimizing the probability of error. In all these algorithms, there is generally a tradeoff between the algorithm performance and the computational complexity. Optimum bit/power loading can be achieved by the well-known water-filling approach. Some loading algorithms can also achieve near-optimal solutions using incremental allocation (e.g. [11-14]). However, the cost in terms of computational complexity associated with both approaches is excessive. To reduce the complexity of bit allocation, closed-form expressions for BER or channel capacity approximations can be exploited. This method, however, requires rounding of the constellation size to integer numbers, hence deviates the allocation from optimality. Wyglinski et al. [11] proposed an optimum bit-loading with reduced complexity. However, the method is still rather computationally complex especially when the number of subcarriers is large, because it includes an extra iterative algorithm to find the initial peak BER in addition to the main algorithm.

In this paper, we attempt to solve the rate maximization problem with target overall BER constraint and uni- 
form power allocation. A simple discrete bit-loading algorithm that approaches the maximum throughput with minimal complexity is presented. The algorithm performance is verified in a widely-accepted power line channel model [16].

\section{Adaptive Bit Loading}

\subsection{Problem Formulation}

The proposed loading algorithm aims to solve the following rate-adaptive problem given a target mean BER $P_{T}$ and a fixed energy distribution across all the subcarriers:

$$
\text { Maximize } \sum_{i=1}^{N} b_{i} \text { subject to } \bar{P}=\frac{\sum_{i=1}^{N} b_{i} P_{i}}{\sum_{i=1}^{N} b_{i}} \leq P_{T}
$$

where $b_{i}$ and $P_{i}$ are the number of bits and BER of the $i$ th subcarrier respectively. $N$ and $\bar{P}$ are the number of used subcarriers and their mean BER respectively. As in other studies, it is assumed that perfect knowledge of the channel gains is available to both the transmitter and the receiver.

Different loading algorithms trying to solve this problem have been discussed in the previous section. These algorithms, however, are either too complex or do not achieve maximum throughput.

\subsection{Proposed Loading Algorithm}

The BER of square MQAM with Gray bit mapping can be approximated [1]:

$$
P_{i}=0.2 \exp \left\{-\frac{1.6 \gamma_{i}}{2^{b_{i}}-1}\right\}, \quad i=1,2, \cdots, N
$$

where $\gamma_{i}=\frac{p_{i}\left|H_{i}\right|^{2}}{\sigma_{i}^{2}}$ is the $i$ th subchannel SNR, with $P_{i}$, $H_{i}$ and $\sigma_{i}^{2}$ being the signal power, channel gain and noise power of the $i$ th subcarrier respectively. Accordingly, the number of bits that can be carried in subchannel $i$ is given by:

$$
b_{i}=\log _{2}\left\{1+\frac{\gamma_{i}}{\Gamma_{i}}\right\}, \quad i=1,2, \cdots, N
$$

where $\Gamma_{i}$ is the SNR gap representing how far the system is from achieving capacity and can be defined from (2) and (3) as:

$$
\Gamma_{i}=-\frac{\ln \left(5 \cdot P_{i}\right)}{1.6}, \quad i=1,2, \cdots, N
$$

The average number of bits per subcarrier in one
OFDM symbol can then be written as:

$$
\begin{aligned}
\bar{b} & =\left(\frac{1}{N}\right) \sum_{i=1}^{N} \log _{2}\left\{1-\frac{1.6 \gamma_{i}}{\ln \left(5 \cdot P_{i}\right)}\right\} \\
& =\left(\frac{1}{N}\right) \sum_{i=1}^{N} \log _{2}\left\{\prod_{i=1}^{N}\left(1-\frac{1.6 \gamma_{i}}{\ln \left(5 \cdot P_{i}\right)}\right)\right\} \\
& =\log _{2}\left\{1-\frac{1.6 \gamma_{m c}}{\ln (5 \cdot \bar{P})}\right\}
\end{aligned}
$$

where $\gamma_{m c}$ is the multichannel SNR which characterizes the set of $N$ subchannels by an equivalent single AWGN that achieves the same data rate with the same error probability [17]. Figure 1 illustrates the concept of the multichannel SNR. From (5), the multichannel SNR can be defined by:

$$
\gamma_{m c}=\frac{\ln \left(5 \cdot P_{i}\right)}{1.6}\left\{1-\left[\prod_{i=1}^{N}\left(1-\frac{1.6 \gamma_{i}}{\ln \left(5 \cdot P_{i}\right)}\right)\right]^{(1 / N)}\right\}
$$

The proposed algorithm initially computes for each subcarrier the maximum number of bits $b_{i}$ that gives a value of $P_{i}$ below $P_{T}$. The resulting overall BER $\bar{P}$ will generally be below $P_{T}$ by a large margin. To exploit this margin, the algorithm then computes the number of extra bits that can be added to the OFDM symbol without violating the BER constraint $P_{T}$.The extra bits are added to the subcarriers that will have the minimum effect in the overall BER. This is done by evaluating $\Delta P$ for each subcarrier where

$$
\Delta P_{i}=b_{i}\left(P_{i}^{+}-P_{i}\right)
$$

and $P_{i}^{+}$is the BER of the $i$ th subchannel when the constellation size is shifted to the immediately higher constellation.

The proposed algorithm takes the following steps:

1) Given SNR values $\gamma_{i}$, find the largest signal constellation $b_{i}$ for each subcarrier for which $P_{i}$ is below $P_{T}$.

2) Calculate the current values of $\bar{b}$ and $\bar{P}$ and com-

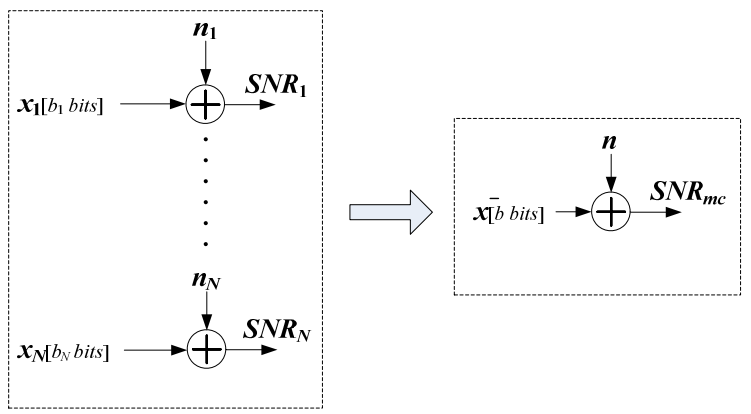

Figure 1. The conceprt of the multichannel SNR [17]. 
pute the multichannel SNR $\gamma_{m c}$ using (6).

3) Use $\gamma_{m c}$ to find the maximum average number of bits $\bar{b}_{\max }$ per subcarrier that satisfies $P_{T}$ :

$$
\bar{b}_{\max }=\log _{2}\left\{1-\frac{1 \cdot 6 \cdot \gamma_{m c}}{\ln \left(5 \cdot P_{T}\right)}\right\}
$$

4) Find the number of extra bits $I=N \cdot\left(\bar{b}_{\max }-\bar{b}\right)$ that can be added to the OFDM symbol.

5) Calculate $\Delta P_{i}$ for all subchannels that have $b_{i}$ below the maximum constellation size. Sort subchannels according to their $\Delta P_{i}$ in increasing order.

Add $I$ extra bits to the subchannels that have the lowest $\Delta P_{i}$ by shifting their constellation to the immediately higher size.

\section{Simulation Results}

\subsection{Channel Model}

Power line networks differ significantly in topology, structure, and physical properties from conventional communication channels such as twisted pair, coaxial, or fiber-optic cables [16]. Because they were not specifically designed for data transmission, power lines provide a harsh environment for higher frequency communication signals. Zimmermann and Dostert [16] proposed a practical channel model that is suitable for describing the transmission behavior of power line channels. The model is based on practical measurements of actual power line networks and is given by the channel transfer function:

$$
H(f)=\sum_{i=1}^{N_{p}} \underbrace{c_{i}}_{\begin{array}{c}
\text { weighting } \\
\text { factor }
\end{array}} \cdot \underbrace{\mathrm{e}^{-\left(a_{0}+a_{1} f^{k}\right) d_{i}}}_{\begin{array}{c}
\text { attenuation } \\
\text { portion }
\end{array}} \cdot \underbrace{\mathrm{e}^{-j 2 \pi f\left(d_{i} / v_{p}\right)}}_{\begin{array}{c}
\text { delay } \\
\text { portion }
\end{array}}
$$

where $N_{p}$ is the number of multipaths, $c_{i}$ and $d_{i}$ are the weighting factor and length of the $i$ th path respectively. Frequency-dependant attenuation is modeled by the parameters $a_{0}, a_{1}$ and $k$.

In the model, the first exponential presents attenuation in the PLC channel, whereas the second exponential, with the propagation speed $v_{p}$, describes the echo scenario. This PLC multipath channel model is used here where parameters of the 15-path channel are given in [16]. Based on this power line channel model, the subchannel gain versus the subchannel index is illustrated in Figure 2 for 1024 subcarriers.

\subsection{Results}

The performance of the proposed bit-loading algorithm is evaluated by computer simulations against incremental and equal-BER loading algorithms based on the achieved

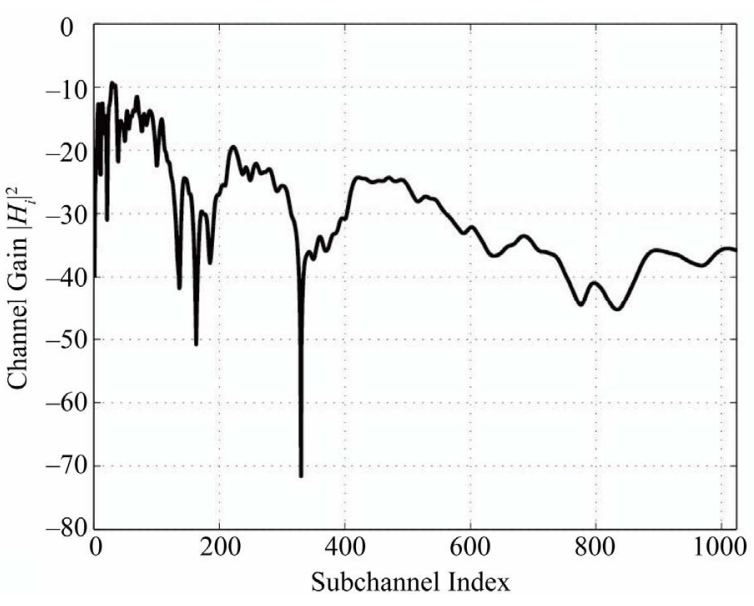

Figure 2. Channel gain for the 15-path power line channel.

average number of bits per subchannel. In incremental loading, all subcarriers are initially allocated the maximum signal constellation and then bits are incrementally removed from the subcarrier with the worst BER until the overall BER satisfies $P_{T}$. In equal-BER allocation, a constant BER threshold (i.e., $P_{T}$ ) is set for all subcarriers and each subcarrier is allocated the maximum number of bits for which its $P_{i}$ is below $P_{T}$. In all the algorithms the system employs OFDM with 1024 subcarriers in the frequency band $1.8-30 \mathrm{MHz}$ and the approximation (2) is used. Each subchannel can be assigned to carry a maximum number of 10 bits.

Figure 3 shows the number of bits allocated to each subcarrier using the proposed algorithm when the target BER constraint $P_{T}$ is equal to $10^{-5}$. The performance of the proposed algorithm, measured in average number of bits per subchannel, is depicted in Figure $\mathbf{4}$ and compared to incremental and equal-BER loading algorithms for two different values of target BER $\left(P_{T}=10^{-3}, 10^{-5}\right)$. The figure shows that the proposed algorithm and incremental loading have similar performance, whereas the

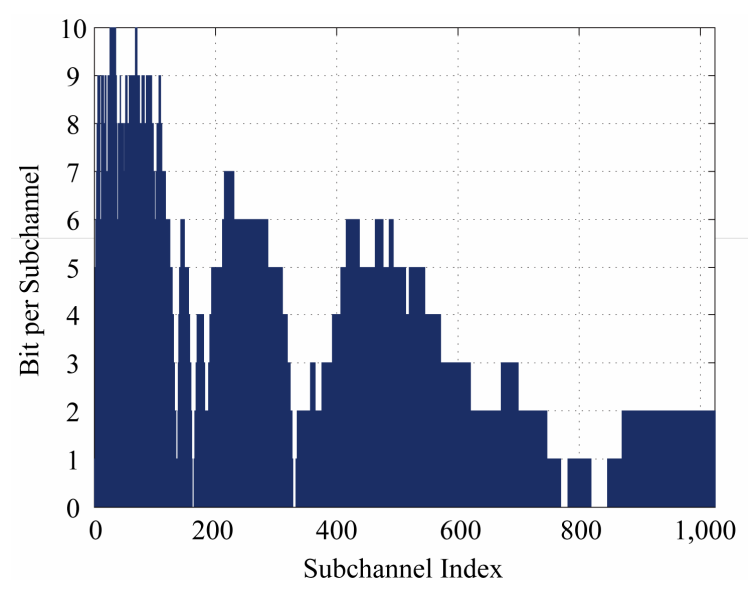

Figure 3. Bit allocation based on the proposed algorithm. 


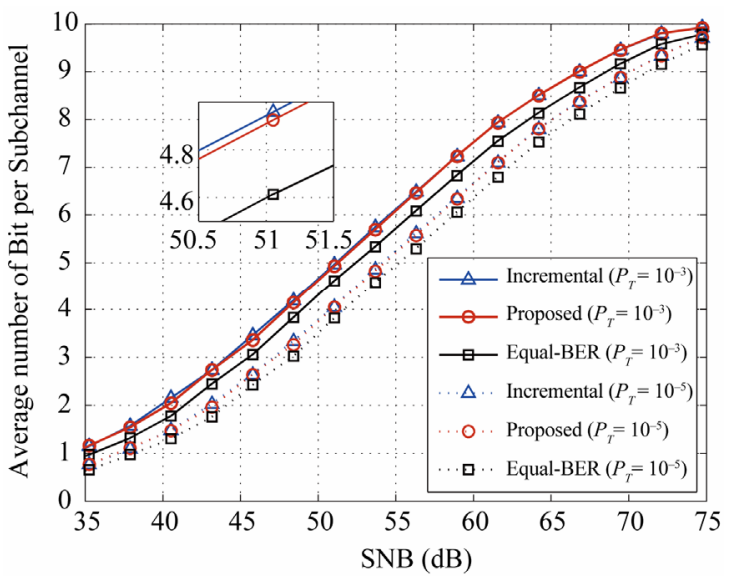

Figure 4. Performance of the proposed loading algorithm as compared to incremental and default loading methods in the 15-path power line channel.

Table1. Mean Computation times (ms) for different values of SNR (CPU: Intel(R) Core(TM)2 Duo 3 GHz ).

\begin{tabular}{ccccc}
\hline \multirow{2}{*}{ Algorithm } & \multicolumn{4}{c}{ SNR Values } \\
\cline { 2 - 5 } & $40 d B$ & $50 d B$ & $60 d B$ & $70 d B$ \\
\hline Incremental & 2.80 & 2.90 & 3.30 & 3.10 \\
Equal-BER & 0.30 & 0.33 & 0.38 & 0.40 \\
Proposed & 0.60 & 0.65 & 0.74 & 0.76 \\
\hline
\end{tabular}

equal-BER loading achieves lower rates. To compare the computational complexity of these algorithms, the computation time (in milli-seconds) taken by each algorithm to reach the final allocation is measured. To insure fairness, each of those algorithms was implemented using Matlab and executed 100 times in the same workstation.

Table 1 illustrates the mean computation times for a 1024-subcarrier OFDM system with $P_{T}$ of $10^{-5}$ for different values of SNR. It should be noted that the proposed algorithm is non-iterative, which results in a significant reduction in algorithm computational com- plexity as compared to the iterative incremental loading. Although the computation time of the proposed algorithm is less than a quarter of that of the incremental loading, both of these algorithms have indistinguishable performances in terms of average number of bits per subchannel. The proposed method takes about twice the time taken by the equal-BER to reach the final allocation. However, it achieves a considerable improvement of more than 300 bits per OFDM symbol over the equalBER loading method.

\section{Conclusions}

This paper presents a simple non-iterative discrete bit-loading algorithm striving to maximize the data rate subject to target BER constraint and uniform power dis- tribution. The algorithm was tested in a practicallyproven power line communication channel model using computer simulations. Results show that the proposed algorithm improves the data rates achieved by the equal-BER loading with a small cost in complexity. When compared to the incremental loading, the proposed algorithm achieves similar rates, but with much lowers computational complexity.

\section{References}

[1] S. T. Chung and A. J. Goldsmith, "Degrees of Freedom in Adaptive Modulation: A Unified View," IEEE Transactions on Communications, Vol. 49, No. 9, 2001, pp. 1561-1571. doi:10.1109/26.950343

[2] P. S. Chow, J. M. Cioffi and J. A. C. Bingham, “A Practical Discrete Multitone Transceiver Loading Algorithm for Data Transmission over Spectrally Shaped Channels," IEEE Transactions on Communications, Vol. 43, No. 234, 2002, pp. 773-775.

[3] Y. George and O. Amrani, "Bit Loading Algorithms for OFDM," Proceedings of the International Symposium on Information Theory (ISIT 2004), Chicago, 2 July 2004, p. 391. doi:10.1109/ISIT.2004.1365428

[4] D. Daly, C. Heneghan and A. D. Fagan, "Power- and Bit-Loading Algorithms for Multitone System," Proceedings of the 3rd International Symposium on Image and Signal Processing and Analysis, Rome, 18-20 September 2003, pp. 639-644. doi:10.1109/ISPA.2003.1296355

[5] H. Rohling and C. Fellenberg, "Successive Bit Loading Scheme,” Electronics Letters, Vol. 45, No. 1, 2009, pp. 214-216. doi:10.1049/el:20093315

[6] E. Guerrini, L. Guerrieri, D. Veronesi, P. Bisaglia and R. Cappelletti, "LLR-Based Bit Loading Algorithm and its Applications to HomPlug AV over OPERA Power-Line Channels with Impulsive Noise," Journal of Communications, Vol. 4, No. 7, 2009, pp. 454-462.

[7] S. Nader-Esfahani and M. Afrasiabi, "Simple Bit Loading Algorithm for OFDM-Based Systems," IET Communications, Vol. 1, No. 3, 2007, pp. 312-316.

[8] K. Lui, B. Tang and Y. Lui, "Adaptive Power Loading Based on Unequal-BER Strategy for OFDM Systems," IEEE Communications Letters, Vol. 13, No. 7, 2009, pp. 474-476. doi:10.1109/LCOMM.2009.082158

[9] K. S. Al-Mawali, F. S. Al-Qahani and Z. M. Hussain, "Adaptive Power Loading for OFDM-Based Power Line Communications Impaired by Impulsive Noise," Proceedings of the IEEE International Symposium on Power Line Communications and its Applications (IEEE ISPLC 2010), Rio de Janeiro, 28-31 March 2010, pp. 78-182. doi:10.1109/ISPLC.2010.5479924

[10] A. Leke and J. M. Cioffi, "A Maximum Rate Loading Algorithm for Discrete Multitone Modulation Systems," Proceedings of IEEE GLOBECOM'97, Phoenix, 3-8 November 1997, pp. 1514-1518. doi:10.1109/GLOCOM.1997.644387 
[11] A. M. Wyglinski, F. Lebeau and P. Kabal, "Bit Loading with BER-Constraint for Multicarrier Systems," IEEE Transactions on Wireless Communications, Vol. 4, No. 4, 2005, pp. 1383-1387. doi:10.1109/TWC.2005.850313

[12] L. Goldfeld, V. Lyandres and D. Wulich, "Minimum BER Power Loading for OFDM in Fading Channel," IEEE Transactions on Communications, Vol. 50, No. 11, 2002, pp. 1729-1733. doi:10.1109/TCOMM.2002.805271

[13] A. M. Wyglinski, F. Labeau and P. Kabal, "An Efficient Bit Allocation Algorithm for Multicarrier Modulation,” Proceedings of the 2004 IEEE Wireless Communications and Networking Conference (IEEE WCNC 2004), Atlanta, 21-25 March 2004, pp. 1194-1199. doi:10.1109/WCNC.2004.1311358

[14] D. Hugh-Hartog, "Ensemble Modem Structure for Im- perfect Transmission Media,” U.S. Patent No. 4,679,227 (July 1987), 4,731,816 (March 1988) and 4,833,706 (May 1989).

[15] J. S. Chow, J. C. Tu and J. M. Cioffi, "A Discrete Multitone Transceiver System for HDSL Applications,” IEEE Journal on Selected Areas in Communications, Vol. 9, No. 6, 1991, pp. 895-908. doi:10.1109/49.93100

[16] M. Zimmermann and K. Dostert, "A Multipath Model for the Powerline Channel," IEEE Transactions on Communications, Vol. 50, No. 4, 2002, pp. 553-559. doi:10.1109/26.996069

[17] J. M. Cioffi, "Chapter 4: Multi-Channel Modulation,” Stanford University website. http://www.stanford.edu/group/cioffi/book/chap4.pdf [09 Jan. 2011] 